\title{
Vortex-antivortex nucleation in superconducting films with arrays of in-plane dipoles
}

\author{
M. V. Milošević* and F. M. Peeters \\ Departement Fysica, Universiteit Antwerpen, Groenenborgerlaan 171, B-2020 Antwerpen, Belgium
}

\begin{abstract}
Using the phenomenological Ginzburg-Landau (GL) theory, we investigate the superconducting (SC) state of a thin SC film under a square array of rectangular ferromagnets (FM) with in-plane magnetization. The equilibrium vortex states consist of vortex-antivortex (VAV) pairs formed under the poles of each magnet due to their specific inhomogeneous magnetic field which makes the total flux penetrating the SC equal zero. Even for a weakly magnetized FM, the nucleation of VAV structures can be stimulated by applying electric current perpendicular to the FM-moment. When the sample is exposed to an additional homogeneous magnetic field $H$, the presence of VAV pairs results in an even $j_{c}(H)$ characteristic, with maximal critical current achieved at $H= \pm H_{\max } \neq 0$, where $H_{\max }$ can be temperature dependent.
\end{abstract}

Key words: Magnetic dipole; vortex-antivortex; lattice configurations.

PACS: 74.78.Na, 74.25.Dw, 74.25.Op, 74.25.Sv

\section{Introduction}

The interaction between superconductivity and magnetism has become one of the most interesting study objects in the last decade. To investigate the effects due to the interplay of the superconducting (SC) order parameter and the non-homogeneous magnetic field resulting from the close-by placed ferromagnets (FMs), several experimental groups fabricated periodic arrays of magnetic dots above or under the superconducting film [1-4]. The main emphasis was placed on the critical current enhancement as the magnets act as trapping centra

\footnotetext{
* Corresponding author.

Email addresses: Milorad.Milosevic@ua.ac.be (M. V. Milošević), Francois.Peeters@ua.ac.be (F. M. Peeters).
}

for the vortices present in the sample. Recently, the interest shifted more towards the perturbation of the superconducting order parameter under the influence of the stray field of the magnets. Fascinating vortex-antivortex (VAV) structures have been predicted theoretically [5-7], and several salient phenomena following from the presence of VAVs were observed experimentally, such as field-polarity and magnetization dependent pinning [8,9], magnetic field-induced superconductivity $[10,11]$, field-shifted maximum of the critical current $[2,11]$, and novel dynamical regimes in SCFM samples $[12,13]$.

In this article, we report an analysis of the the superconducting state of a thin superconducting film nanostructured on top with a rectangular array of 


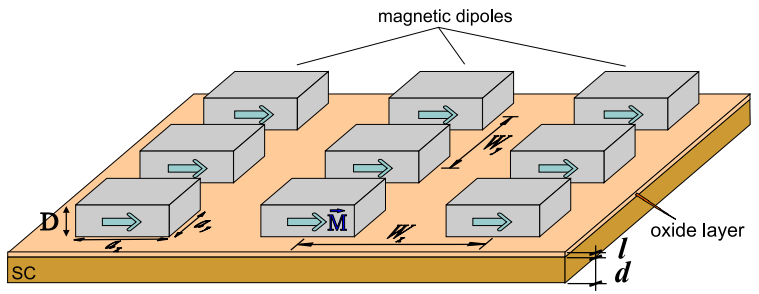

Fig. 1. [Color online] Schematic view of a superconducting film (with thickness $d$ ) covered by an oxide layer (thickness $l$ ), under a regular array (periodicity $W_{x}, W_{y}$ ) of rectangular magnetic dipoles (with size $a_{x} \times a_{y}$, thickness $D$, and in-plane magnetization $M$ ).

in-plane dipoles (see Fig. 1), with/without presence of additional external magnetic field and/or electric current. We study the vortex configurations and critical phenomena within the framework of the non-linear Ginzburg-Landau (GL) theory.

\section{Theoretical formalism}

For thin superconductors ( $d$ smaller than characteristic lengths $\xi$ and $\lambda$ ), it is allowed to assume that the order parameter is uniform along the film thickness, which reduces the GL equations to

$$
\begin{aligned}
& \left(-i \vec{\nabla}_{2 D}-\vec{A}\right)^{2} \psi=\psi\left(1-|\psi|^{2}\right), \\
& -\Delta_{3 D} \vec{A}=\frac{d}{\kappa^{2}} \delta(z) \vec{j}_{2 D},
\end{aligned}
$$

where

$\vec{j}_{2 D}=\frac{1}{2 i}\left(\psi^{*} \vec{\nabla}_{2 D} \psi-\psi \vec{\nabla}_{2 D} \psi^{*}\right)-|\psi|^{2} \vec{A}$,

is the density of the superconducting current and $\vec{A}$ is the local vector potential (resulting from all applied drives and the supercurrents). Here, all distances are expressed in units of the coherence length $\xi$, the vector potential in $c \hbar / 2 e \xi$, and the magnetic field in $H_{c 2}=c \hbar / 2 e \xi^{2}=\kappa \sqrt{2} H_{c}, \kappa$ being the GL parameter. To find the self-consistent solution of Eqs. (1) and (2) for the order parameter we use an iteration procedure based on the GaussSeidel technique. The vector potential is obtained with the fast Fourier transform technique. The details of this numerical approach are explained in Ref. [14]. We use periodic boundary conditions [15] in order to simulate an infinite superconducting film. These boundary conditions result in a periodic repetition of not only the superconductor but the magnetic structures as well (included in the simulation via their stray field). In the cases when an additional homogeneous field is applied, two conditions must be met: (i) the flux quantization per simulation region following from the virial theorem [15], and (ii) the gauge transformation must be properly chosen, as explained in Ref. [6]. The addition of a DC current to this formalism is realized by an added constant to the initially applied vector potential. We assume that critical current of the sample is reached when the stationary solution of Eqs. (1-2) can no longer be found (e.g. motion of vortices can no longer be prevented).

\section{The vortex-antivortex coexistence}

Throughout the paper, we will consider $\mathrm{a} \mathrm{Pb}$ film of thickness $d=50 \mathrm{~nm}, \xi(0)=40 \mathrm{~nm}$, and $\kappa=1.2$, covered with an oxide layer of thickness $l=20 \mathrm{~nm}$, and an array of magnetic dipoles $\left[W_{x}=\right.$ $2 \mu \mathrm{m}, W_{y}=1 \mu \mathrm{m}, D=100 \mathrm{~nm}, a_{x}, a_{y}$ and $M$ may vary (see Fig. 1)]. In this section, no external drives are applied, and the magnetic dipoles are the only source of magnetic field. To find the different vortex configurations, which include the metastable states, we search for the lowest energy solutions of Eqs. (1) and (2) starting from different randomly generated initial configurations. Then we increase/decrease slowly the magnetization of the magnetic dots $M$ and recalculate each time the exact vortex structure. By comparing the Gibbs free energies (see e.g. Ref. [14]) of the different vortex configurations we find the ground state as the state with the lowest energy. The ground state free energy of the considered system as a function of magnetization is shown in Fig. 2 for different sizes of the magnetic dipoles, namely $a_{x} \times a_{y}=0.6 \mu \mathrm{m} \times 0.2 \mu \mathrm{m}$, $0.6 \mu \mathrm{m} \times 0.4 \mu \mathrm{m}, 1.0 \mu \mathrm{m} \times 0.4 \mu \mathrm{m}$, and $1.5 \mu \mathrm{m} \times 0.4 \mu \mathrm{m}$, at temperature $T / T_{c}=0.9$.

Knowing the magnetic field profile of the inplane dipole, each dipole favors the nucleation of the vortex-antivortex (VAV) pairs at its poles. Lattice of individual VAV pairs has already been 

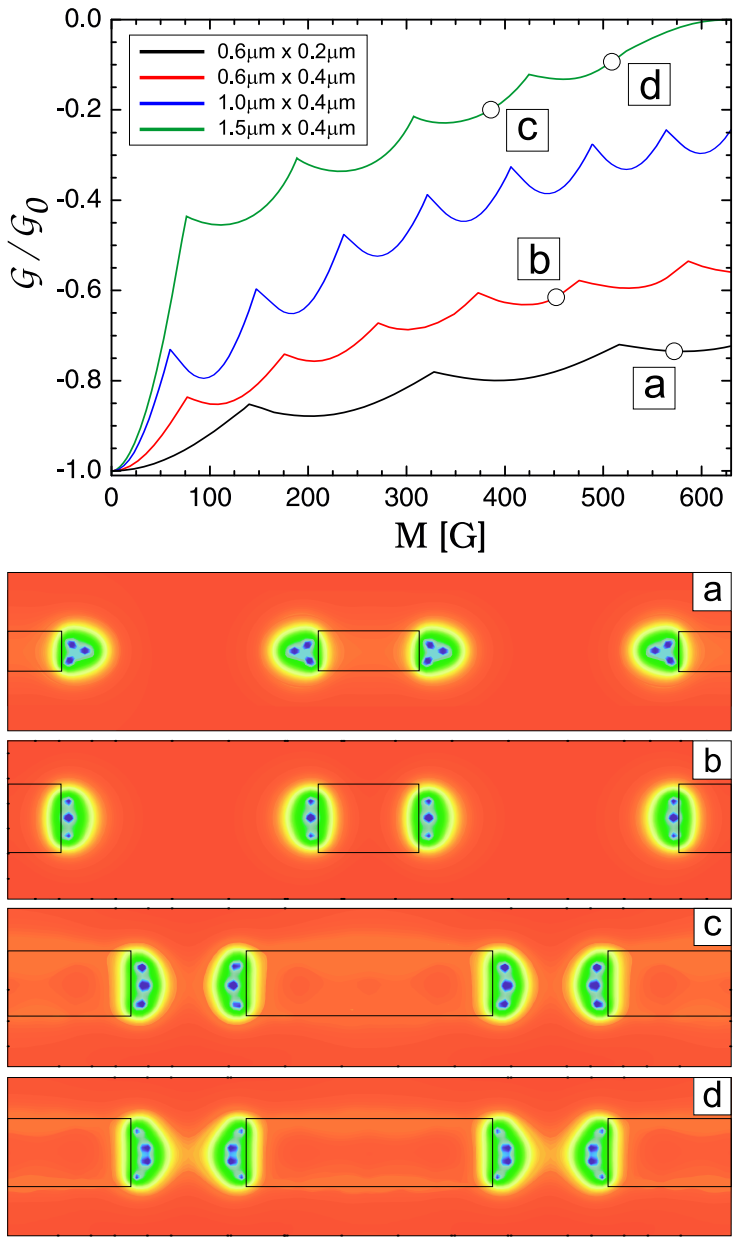

Fig. 2. [Color online] The Gibbs free energy of a $\mathrm{Pb}$ film $\left(d=50 \mathrm{~nm}, \xi(0)=40 \mathrm{~nm}, T=0.9 T_{c}, \kappa=1.2\right)$ with a regular array $\left(W_{x}=2 \mu \mathrm{m}, W_{y}=1 \mu \mathrm{m}\right)$ of in-plane magnetic dipoles $(D=100 \mathrm{~nm}, l=20 \mathrm{~nm})$ on top. Figs. (a-d) show the Cooper-pair density (logarithmic scale, blue/red-low/high density) of the vortex-antivortex states indicated by open dots in the free-energy diagram (in the area $4 \mu \mathrm{m} \times 1 \mu \mathrm{m}$, solid lines denote the edges of the magnets).

observed experimentally using the Scanning HallProbe Microscopy (see e.g. Ref. [8]). However, an interesting question arises: what will happen for larger vorticity? Namely, the stray field of the dipole has a maximum in a single point at its pole, and one expects the nucleation of a giant-vortex, while the open geometry of the superconducting film favors individual vortices. This competition results in different VAV configurations, some of which shown as the Cooper-pair density contourplots in Fig. 2(a-d). For example, note that in Fig. 2(a) three vortices (analogously for antivortices) form an equilateral triangle around the field-maximum, since the magnet is really dipolelike $\left(a_{y} \approx 1.6 \xi\right)$. However, once the width of the magnet is enlarged, the maximal stray field is distributed more evenly along $a_{y}$, and the vortices prefer to sit on that line [Fig. 2(b)]. Obviously, after a certain number of nucleated VAV pairs is reached, the second line of vortices (or a more complicated structure) may be formed. This saturation number will depend on the size of the magnet and its magnetization [5]. The exact vortex-antivortex configuration at the poles of the in-plane magnet as a function of its size and shape is also important due to the fact that an in-plane magnet profoundly mimics the magnetic film with a single domain-wall [16] and these results can serve for understanding of vortex-antivortex behavior around such a wall.

However, it is not only $a_{y}$ that affects the vortex structure. Changing $a_{x}$ makes significant difference, not only because the quantity of magnetic material increases (and consequently the stray field for fixed magnetization), but also because neighboring dipoles in the lattice come in close proximity (for period $W_{x}, W_{y}$ fixed). This can be already seen from Fig. 2(c), where 3 vortices at one pole of the magnet are clearly attracted by the antivortices induced by the adjacent magnet. This attraction partly follows from the annihilation of induced currents in the sample, maximal in the central region. This compensation of induced currents (and stray fields) becomes more pronounced as $a_{x}$ increases. So for $a_{x}=1.5 \mu \mathrm{m}$ (see Fig. 2), vorticity larger than 4 at the poles becomes impossible (even for large $M$ ), since newly induced vortices annihilate with antivortices from the neighboring site. This dynamical process of nucleation and annihilation can be ended only by an exit to a normal state. Note that in the latter case, due to the stray field compensation between neighboring magnets, the $\Delta M$ necessary for a VAV nucleation increases (see free energy in Fig. 2), which is another important influence of a lattice geometry. 


\section{The response to applied current}

In what follows, we discuss the influence of a DC current applied to the sample in the $y$-direction (therefore perpendicularly to the magnetic moment of the dipoles). Two cases will be considered, namely the previous one, with magnetic dots as only field source, and the case when an applied homogeneous magnetic field is present in addition to the dipoles.

\subsection{Stimulated vortex-antivortex nucleation}

In order to properly understand the influence of an applied current, one must consider first the currents present in the sample. Fig. 3(a) shows the contourplot and the vectorplot of the Meissner currents induced in the film by the in-plane magnet (one unit cell is shown). One should note the whirling flow of currents around the poles of the magnet, where the stray field has opposite polarity. Due to the proximity of the neighboring magnets, this current profile effectively results in two oppo-
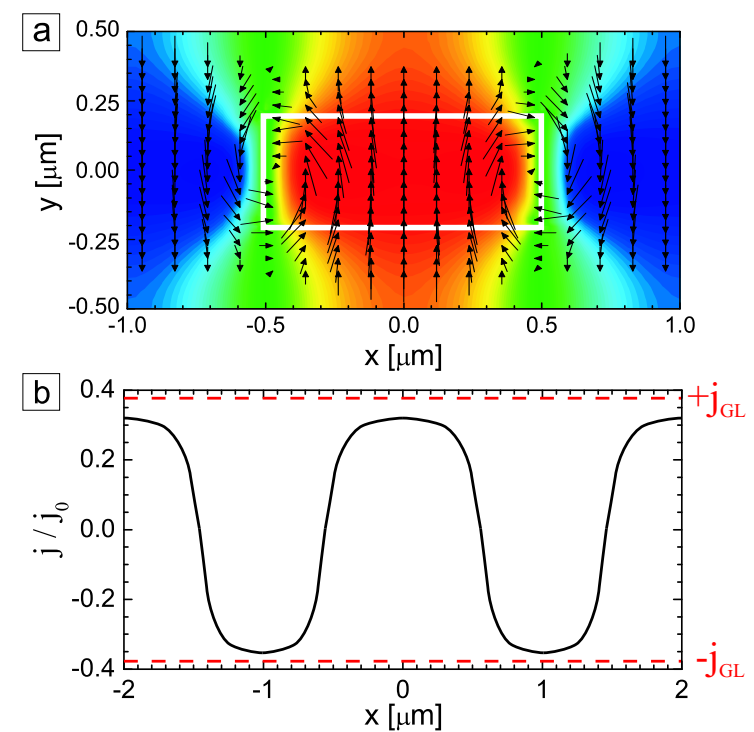

Fig. 3. [Color online] (a) The vectorplot of the Meissner current superimposed on its contourplot (blue/red-low/high density), induced by $1 \mu \mathrm{m} \times 0.4 \mu \mathrm{m} \times 0.1 \mu \mathrm{m}$ magnets with $M=50 \mathrm{G}$ (denoted by white lines, see also Fig. 2); (b) the current profile along the $y=0$ line (in units of $\left.j_{0}=c H_{c 2} \xi / 4 \pi \lambda^{2}\right)$. site current flows, perpendicularly to the magnetic moment, under and aside the magnet, respectively. The magnitude of current across the sample (in $y=$ 0 direction) is plotted in Fig. 3(b). We found that for chosen parameters, the negative amplitude of the current between the dipoles is larger than the positive one under the dipoles. This follows from the proximity of the magnets in a lattice, and will always be the case when $W_{x}<2 a_{x}$ (total current in the sample must be conserved and therefore must be zero). The important consequence of this feature is that the vortex-antivortex nucleation in SC films under such magnetic lattices will occur between the magnets, not under them as found in the case of a single in-plane magnet (and $W_{x}>2 a_{x}$ ) [9].

Fig. 3(b) also shows the magnitude of the Meissner currents compared to the depairing (or Ginzburg-Landau) current $j_{G L}$, generally regarded as the threshold current for the vortex (in our case vortex-antivortex) nucleation. As can be easily deducted from the figure and the previous discussion, applied external current $j$ in $y$-direction will cause nucleation of VAV pairs, for both its polarities. Therefore, for positive $j$, VAV pairs dissociate under the magnets, whereas for negative $j$ the nucleation occurs between the magnets. Interestingly enough, both processes result in the same vortex-antivortex configurations (vortices under positive pole and antivortices under negative pole of each magnet).

When external current is added, the magnetsinduced currents are compensated in some part of the sample. This compensation effect is analogous to the magnetic field compensation in SC films with arrays of out-of-plane magnets in external field, leading to the field-induced-superconductivity [10]. However, in our case, while compensating currents in one part of the sample we are enhancing them in the other, causing VAV nucleation. These VAVs are not confined, they move across the sample in opposite directions and annihilate. This dynamics destroys superconductivity and although the idea seems fine, the so-called currentinduced-superconductivity cannot be realized. 
4.2. Critical current vs. applied homogeneous magnetic field

One of the first applications of arrays of magnetic dots in combination with superconducting films was to pin the external flux lines piercing through the film at a given magnetic field. This pinning results in a reduced vortex motion, and consequently enhances the critical current. As the first experiment of this kind, regular arrays of in-plane dipoles were used in Ref. [1]. The pronounced peaklike maxima in critical current were found at the so-called matching fields, where the number of vortices 'matched' an integer multiple of the number of pinning sites (i.e. magnets). The $j_{c}(H)$ characteristics showed symmetric behavior with respect to the polarity of the applied field (with a maximum for $H=0$ ), as the vortex pinning occurs on opposite poles of each magnet [9].

However, in this experiment, the stray field of the magnets was not sufficient for the vortexantivortex nucleation, and all vortices present in the sample were induced by the external homogenous field. The question of pinning in the presence of magnet-induced vortex-antivortex pairs is a complex one and is discussed in detail in Refs. $[8,9]$. If one VAV pair is induced by each magnet, in a most likely scenario, the external vortex is attracted and annihilated by an existing antivortex (though this behavior depends on other parameters as well). Our aim here is to investigate the behavior of the critical current as a function of applied homogeneous magnetic field when magnetization of the dipoles is large. For this purpose, we calculated the critical current of the sample with dipoles of dimensions $0.6 \mu \mathrm{m} \times 0.2 \mu \mathrm{m}$, thickness $D=100 \mathrm{~nm}$, and magnetization $M=400 \mathrm{G}$, for the values of the external magnetic field providing an integer number of flux quanta in a $2 \times 2$ simulation region. The results are shown in Fig. 4, for temperatures $0.9 T_{c}, 0.95 T_{c}$, and $0.98 T_{c}$.

As a most salient result, we found a maximum of the critical current at the second matching field, not for $H=0$ as found in Ref. [1]. From Fig. 2 (bottom curve) one notes that for chosen parameters each magnet induces two vortex-antivortex pairs in the sample, which leads to the maximal com-

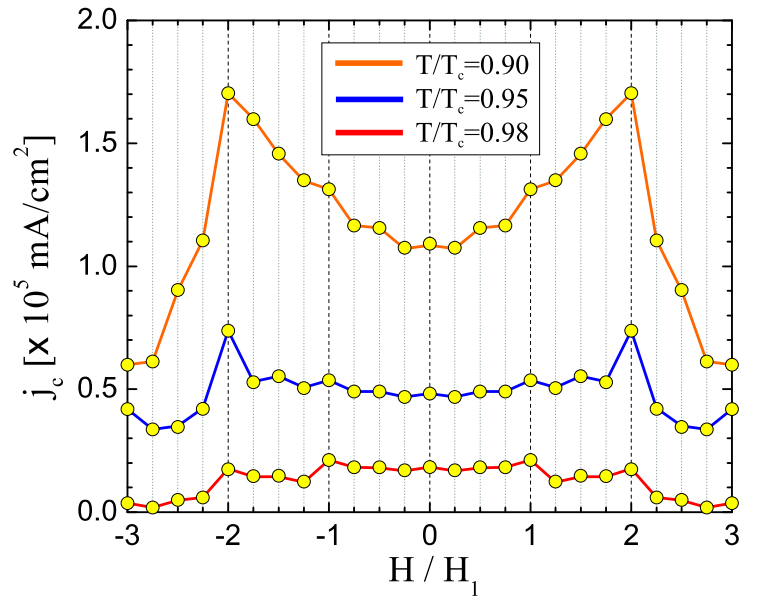

Fig. 4. [Color online] The applied current versus magnetic field (in units of the first matching field) superconducting-normal phase boundary for the sample corresponding to the black curve in Fig. 2 and $M=400 \mathrm{G}$, at temperatures $0.9 T_{c}, 0.95 T_{c}$, and $0.98 T_{c}$ (top to bottom).

pensation between the existing antivortices and added external flux lines for $H=H_{2}$. This behavior is analogous to the case of out-of-plane magnets, where a field-shifted-maximum of the critical current was predicted [11]. Few differences should be noted. First, for in-plane dipoles, the $j_{c}(H)$ curve is always symmetric with respect to $H=$ 0 . Second, a sharp drop in $j_{c}$ is found for $H>$ $H_{2}$. Namely, additional vortices can no longer be pinned by the dipoles, and occupy interstitial sites. It is well known that these vortices are more easily subjected to motion than the pinned ones. Also note that for $H<H_{2}$, the criterion for the critical current is not the motion of vortices, but the dynamical process of stimulated nucleation of vortexantivortex pairs, their depinning and annihilation across the sample.

As one more difference from samples with outof-plane pinning arrays, we note that with increasing temperature, the position of the maximum of the critical current shifts towards lower matching fields. The reason is that for $T=0.98 T_{c}$ only one VAV pair per magnetic dipole is present in the sample. This remarkable temperature dependence of the number of VAV pairs can be explained as follows. With increasing temperature the size of the cores of vortices and antivortices increases. Therefore, although magnetization of each magnet 
is sufficient for nucleation of two VAV pairs per dipole, one of the two induced vortices starts to overlap with an antivortex of the adjacent magnet. This interaction leads to annihilation, and only one vortex-antivortex pair per magnet remains. To our knowledge, the behavior of the critical current similar to the one shown in Fig. 4 has been recently observed [17].

\section{Acknowledgements}

This work was supported by the Flemish Science Foundation (FWO-Vl), the Belgian Science Policy (IUAP), the joint JSPS-ESF program on Nanoscience and Engineering in Superconductivity, and the ESF-network Arrays of Quantum Dots and Josephson Junctions.

\section{References}

[1] J. I. Martin, M. Velez, J. Nogues, and I. K. Schuller, Phys. Rev. Lett. 79, 1929 (1997); Y. Jaccard, J. I. Martin, M.-C. Cyrille, M. Velez, J. L. Vicent, and I. K. Schuller, Phys. Rev. B 58, 8232 (1998); J. I. Martin, M. Velez, A. Hoffmann, I. K. Schuller, and J. L. Vicent, Phys. Rev. Lett. 83, 1022 (1999); J. I. Martin, M. Velez, A. Hoffmann, I. K. Schuller, and J. L. Vicent, Phys. Rev. B 62, 9110 (2000).

[2] D. J. Morgan and J. B. Ketterson, Phys. Rev. Lett. 80, 3614 (1998).

[3] M. J. Van Bael, L. Van Look, K. Temst, M. Lange, J. Bekaert, G. Guentherodt, V. V. Moshchalkov, and Y. Bruynseraede, Physica C 332, 12 (2000); M. J. Van Bael, K. Temst, V. V. Moshchalkov, and Y. Bruynseraede, Phys. Rev. B 59, 14674 (1999); M. J. Van Bael, M. Lange, S. Raedts, V. V. Moshchalkov, A. N. Grigorenko, and S. J. Bending, Phys. Rev. B 68, 014509 (2003).

[4] J. E. Villegas, S. Savel'ev, F. Nori, E. M. Gonzalez, J. V. Anguita, R. Garcia, and J. L. Vicent, Science 302, 1188 (2003)

[5] M. V. Milošević and F. M. Peeters, Phys. Rev. B 68, 024509 (2003).

[6] M. V. Milošević and F. M. Peeters, Phys. Rev. Lett. 93, 267006 (2004).

[7] M. V. Milošević and F. M. Peeters, Phys. Rev. Lett. 94, 227001 (2005).
[8] M. J. Van Bael, J. Bekaert, K. Temst, L. Van Look, V. V. Moshchalkov, Y. Bruynseraede, G. D. Howells, A. N. Grigorenko, S. J. Bending, and G. Borghs, Phys. Rev. Lett. 86, 155 (2001).

[9] M. V. Milošević and F. M. Peeters, Phys. Rev. B 69, 104522 (2004).

[10] M. Lange, M. J. Van Bael, Y. Bruynseraede, and V. V. Moshchalkov, Phys. Rev. Lett. 90, 197006 (2003).

[11] M. V. Milošević and F. M. Peeters, Europhys. Lett. 70, 670 (2005).

[12] M. Lange, M. J. Van Bael, A. V. Silhanek, and V. V. Moshchalkov, Phys. Rev. B 72, 052507 (2005).

[13] M. V. Milošević and F. M. Peeters, Phys. Rev. Lett. 95, 147004 (2005).

[14] V. A. Schweigert and F. M. Peeters, Phys. Rev. B 57, 13817 (1998).

[15] M. M. Doria, J. E. Gubernatis, and D. Rainer, Phys. Rev. B 39, 9573 (1989).

[16] A. Yu. Aladyshkin, A. I. Buzdin, A. A. Fraerman, A. S. Melnikov, D. A. Ryzhov, and A. V. Sokolov, Phys. Rev. B. 68, 184508 (2003); Z. R. Yang, M. Lange, A. Volodin, R. Szymczak, and V. V. Moshchalkov, Nature Mat. 3, 793 (2004).

[17] F.G. Aliev, private communication. 